\title{
Dificuldades dos Alunos na Aprendizagem da Lei de Ampère, à Luz da Teoria dos Modelos Mentais de Johnson-Laird
}

\author{
(Students' difficulties in the learning of Ampère's Law, in the light of Johnson-Laird's mental models theory) \\ Marco Antonio Moreira e Adriano de Oliveira Pinto \\ Instituto de Física, UFRGS, C.P. 15051, CEP 91501-970, Campus do Vale, Porto Alegre, RS \\ e-mail:moreira@if.ufrgs.br; e-mail: adriano@if.ufrgs.br
}

Recebido em 28 de maio, 2003. Aceito em 30 de setembro, 2003.

\begin{abstract}
Este trabalho procurou detectar quais as dificuldades mais freqüentes que alunos de Física Geral têm na aprendizagem da Lei de Ampère, visando interpretar as mesmas com base na Teoria dos Modelos Mentais de JohnsonLaird. Foram pesquisados cerca de 230 estudantes, dos cursos de Engenharia e Matemática da UFRGS, ao longo de dois semestres letivos, por meio de suas respostas a questões e problemas envolvendo a Lei de Ampère. Ficou evidente que os alunos resolvem os problemas mecanicamente e respondem a questões descritivas da mesma forma, o que forneceu dados para levantar as dificuldades relacionadas a esse mecanicismo, que se traduzem em interpretações incompletas e/ou equivocadas da Lei de Ampère, objeto de nossa análise.
\end{abstract}

The research reported in this paper attempted to detect the most frequent difficulties students have in the learning of Ampère's Law, trying to interpret them in the light of Johnson-Laird's mental models theory. Some 230 engineering and mathematics students were researched, during two academic semesters, through their answers to conceptual questions and their written solutions to problems involving Ampère's Law. Research findings clearly show that students solve problems mechanically and provide answers to conceptual questions in the same way. This kind of mechanicism that appear in incomplete or wrong interpretations of the law was object of our analysis.

\section{Objetivos e fundamentação teórica}

Esta pesquisa se propõe a identificar possíveis dificuldades que alunos de Física Geral têm na aprendizagem significativa da Lei de Ampère, bem como interpretar essas dificuldades à luz da teoria dos Modelos Mentais de Johnson-Laird (1983).

Nas três últimas décadas, a pesquisa em ensino de Física se revolucionou a cada década: nos anos 70, surgiu o estudo das concepções alternativas (idéias prévias que os alunos possuem, muitas vezes decorrentes de suas experiências pessoais, e que normalmente se encontram incorretas em relação às idéias compartilhadas pela comunidade científica); nos 80, apoiada no estudo citado anteriormente, houve a exploração do tema da mudança conceitual (atitude de superação das concepções alternativas pelas concepções tidas como válidas cientificamente). A década de 90 foi palco da abordagem do tema das representações mentais, com ênfase na linha dos modelos mentais.

As concepções alternativas dos alunos podem ser interpretadas como suas teorias implícitas (representações implícitas geradas por processos cognitivos implícitos, baseados em regras de caráter essencialmente associativo e indutivo, que surgem na tentativa de o aluno compreen- der o mundo; o que, porém, ocorre de maneira implícita, ou seja, sem que a pessoa esteja consciente dos processos que utiliza para fazer essas predições ou de suas próprias representações, e se isto acontece, se dá em um nível muito superficial) e assim sendo, se correspondem mais com modelos mentais do que com representações esquemáticas, explicitamente presentes na memória do sujeito (Pozo, 1999). A mudança conceitual não pode e nem deve implicar na substituição de um tipo de representação por outro (op. cit., p. 516), porém um ensino de Ciência baseado em modelos - de modelos mentais a científicos - pode fazer com que o professor exerça seu papel de mediador do conhecimento (ibidem, p. 518).

Em um primeiro envolvimento com o tema, precisamos definir o que se entende por modelo mental. Para JohnsonLaird (1983) e Gentner e Stevens (1983), modelo mental é o construto psicológico que as pessoas formam na interação com outras pessoas, com o meio ou com algum artefato, de modo que lhes permita avaliar tal interação e predizer o comportamento dos sistemas em relações futuras.

Há várias linhas de estudo das representações mentais na Psicologia Cognitiva atual. Foi nossa opção trabalhar com a teoria de Johnson-Laird (1983), pelo fato de a mesma ser, em nosso julgamento, a mais completa e coerente. 
Assim, todo este trabalho foi norteado pela teoria de Johnson-Laird, de modo que procuramos, sempre que possível, interpretar e explanar os resultados obtidos em termos de representações mentais no sentido das propostas de Johnson-Laird.

Para Johnson-Laird, há três tipos de representações mentais. Representações proposicionais são cadeias de símbolos que correspondem à linguagem natural (são verbalmente expressáveis). Imagens são representações muito específicas que se baseiam sobretudo nos aspectos perceptivos de certos objetos ou eventos, vistos de um certo ângulo. Modelos mentais são análogos estruturais do mundo. Por exemplo, a situação "o prato está na mesa" pode ser representada mentalmente como uma proposição (pois é verbalmente expressável), como um modelo mental (de qualquer prato em qualquer mesa), ou como uma imagem (um certo prato em uma certa mesa). As imagens são vistas dos modelos mentais.

Um modelo mental é uma representação interna de informações que corresponde analogamente com aquilo que está sendo representado (Moreira, 1996). Um modelo mental representa um determinado estado de coisas. Segundo Johnson-Laird, as pessoas raciocinam empregando modelos mentais, os quais podem ser alterados e combinados recursivamente conforme for necessário.

Segundo Norman (1983), modelos conceituais são modelos projetados como instrumentos para a compreensão ou para o ensino de sistemas físicos. Por outro lado, para Johnson-Laird (1983), modelos mentais são modelos que as pessoas constróem para representar estados físicos; tais modelos são análogos estruturais de estados de coisas do mundo; não precisam ser acurados, mas sobretudo devem ser funcionais. De acordo com estas definições, a Lei de Ampère poderia ser interpretada como um modelo conceitual que, para ser aprendido de maneira significativa implicaria a construção de um modelo mental adequado (isto é, um modelo que permitisse descrevê-la, explicá-la e usá-la para fazer previsões).

\section{Metodologia}

Trabalhamos, em uma primeira etapa, com cerca de 80 estudantes dos cursos de Engenharia e Matemática, que cursaram a disciplina Física II - C, do Departamento de Física da UFRGS, durante o primeiro semestre letivo de 1998. Em uma segunda coleta de dados, trabalhamos com aproximadamente 150 estudantes nas mesmas condições da etapa anterior, que realizaram a disciplina no segundo semestre de 1998. Nos dois processos, coletamos suas respostas a questões e problemas envolvendo a Lei de Ampère. Foi peculiar à primeira etapa de coleta, uma interação mais direta com os alunos em situação de sala de aula, devido à estratégia de trabalho em pequenos grupos usada nessa oportunidade, bem como a confecção de um mapa conceitual sobre a Lei de Ampère.

As questões conceituais e problemas envolvendo a Lei de Ampère em questão neste trabalho podem ser especificadas como típicas do livro "Fundamentos de Física", $4^{a}$ edição, de Halliday, Resnick e Walker (1996). A todos os cerca de 230 estudantes com quem trabalhamos, solicitamos que respondessem a uma questão explanatória que serviu como ferramenta de pesquisa aos nossos propósitos de identificar e interpretar as dificuldades dos mesmos com a Lei de Ampère. Esta questão explanatória, a qual chamamos de modo interno a esta pesquisa de questão-chave, tinha por enunciado:

"Escreva a Lei de Ampère na sua forma integral, interprete-a fisicamente e fale sobre ela, isto é, diga tudo o que puder sobre esta lei. Exemplifique sua explicação, faça uso de desenhos ou diagramas se necessário. Diga se você teve dificuldades para entender esta lei e, se for o caso, quais foram essas dificuldades.”

Este trabalho de pesquisa foi permanentemente norteado pela idéia da expressabilidade exterior das representações internas do indivíduo de modo refletido e fidedigno, ou seja, a idéia através da qual cremos que as manifestações representacionais externas do indivíduo (registros escritos e desenhos, no caso de análise à distância, e expressão verbal por meio de perguntas, opiniões e asserções no caso de situação de sala de aula - interação direta) refletem de modo coerente as suas representações mentais internas. Assim, levamos em conta todas as manifestações individuais exteriores no intuito de as mesmas nos fornecerem traços correspondentes à operação mental dos indivíduos em questão. Deste modo procuramos inferir se os alunos modelizam (ou não) a Lei de Ampère, e, mais importante do que isso, que dificuldades têm eles com essa lei.

Discorreremos a seguir sobre a forma como foi apresentado o tópico "Lei de Ampère" aos alunos com os quais se realizou este trabalho. Primeiro, falaremos da forma com que esse tema é apresentado pelo livro didático adotado e, posteriormente, detalharemos como em cada conjunto de turmas foi apresentada pelo respectivo professor tal lei a seus alunos.

O livro tomado por bibliografia básica em todas as seis turmas que participaram da pesquisa é o mesmo: trata-se do conhecido título "Fundamentos de Física", volume 3, de D. Halliday, R. Resnick e J. Walker, $4^{a}$ edição, LTC, 1996.

A Lei de Ampère se situa no capítulo 31 da obra, sendo tal capítulo devotado parcialmente a tal lei. A abordagem é realizada ao longo de sete seções distribuídas no capítulo. Cabe aqui fazer um breve relato de como é apresentado esse tópico em tal capítulo.

$\mathrm{Na}$ quinta seção desse capítulo é que se fala propriamente em Lei de Ampère. A lei é invocada pelo texto como uma das Equações de Maxwell pelo fato de, assim como a Lei de Gauss está para a Lei de Coulomb, a Lei de Ampère está para a Lei de Biot-Savart: a primeira tem uma forma mais compatível com as outras Equações de Maxwell e permite resolver problemas de campo magnético de alto grau de simetria com facilidade e elegância (Halliday et al., 1996, p. 190). Logo a seguir, é apresentada a Lei de Ampère, em sua forma integral, e, na seqüência, esclarecidos os dois termos 
subjacentes a ela que são fundamentais à sua compreensão e estrutura: a curva amperiana e a corrente líquida. Para ilustrar o conceito de corrente líquida, é apresentada uma figura ilustrativa desse conceito. Os autores comentam também sobre o fato de a soma algébrica das correntes englobadas pela amperiana ser computado como o cálculo da corrente líquida, o que é corroborado por uma ilustração que aborda a regra da mão direita adaptada à situação. Imediatamente, o texto aborda o cálculo do campo magnético gerado por um fio retilíneo longo utilizando a Lei de Ampère e compara o resultado com aquele obtido pela Lei de Biot-Savart que, é claro, é o mesmo, porém, segundo os autores, obtido desta feita com menor esforço. Um segundo exemplo se segue: é apresentado o cálculo do campo magnético gerado por uma corrente em um fio retilíneo longo de seção transversal circular, com corrente uniformemente distribuída sobre a seção transversal do fio, para os casos clássicos envolvendo as grandezas $r$ (raio da curva amperiana) e $R$ (raio do fio) nas formas $r<R$ e $r>R$. No caso $r<R$, os autores dão ênfase ao fato de se considerar a corrente englobada pela amperiana (como corrente ponderada).

Sucedem-se então um questionário, que aborda algumas questões conceituais simples, e uma tradicional seção de exercícios e problemas.

Os exercícios e problemas sobre a Lei de Ampère invariavelmente tratam sobre a determinação da expressão para o campo magnético $\mathbf{B}$ em diversas situações que são variações dos casos clássicos (fio condutor, cabo coaxial, solenóide, toróide). Desse modo, é fácil deduzir que os problemas induzem um caráter metódico à Lei de Ampère, atribuindo a ela um método de cálculo do módulo do campo magnético, em abstenção ao caráter fenomenológico que ela possui.

Em relação à forma como a Lei de Ampère foi apresentada a cada conjunto de turmas pelo respectivo professor, faremos um breve relato a seguir:

- Turmas $C$ e $H$ : A respeito da Lei de Ampère (capítulo 31 do livro-texto, que trata basicamente de tal lei, mas também de conceitos associados, como Lei de Biot-Savart, força magnética entre fios paralelos, aplicações da Lei de Ampère, casos do solenóide e do toróide) foram empregadas três aulas expositivas, uma aula de demonstração (pelo professor) de aplicações da Lei de Ampère em problemas, duas aulas de resolução de problemas em aula com os alunos reunidos em grupos com assistência do professor e dos monitores, e uma nova aula de resolução de problemas realizada pelo professor. Foram, portanto, devotadas sete aulas a este capítulo. As aulas expositivas foram enfáticas com a fenomenologia da lei e conceitos associados, refletindo porém os conteúdos do livro-texto de maneira levemente modificada, onde o professor procurou imprimir um caráter pessoal à exposição dos conteúdos. A aula de demonstração de aplicações da lei se deteve na resolução dos casos clássicos, bem como a aula de resolução de problemas nesse fato se restringiu. Quando das aulas de resolução de problemas realizada pelos alunos, um dos autores do presente trabalho teve a oportunidade de interagir com eles e nela detectar características de dificuldades de aprendizagem da Lei de
Ampère que se repetiriam no material coletado posteriormente na prova e a seguir analisado.

- Turmas $M$ e K: O professor destas turmas empregou cinco aulas para abordar o capítulo sobre Lei de Ampère, sob a forma de três aulas expositivas (que refletiam puramente o conteúdo do livro-texto, em uma releitura resumida do mesmo) e duas aulas de resolução de problemas, versando sobre casos clássicos.

- Turmas A e B: Aqui cabe o mesmo comentário aplicado às aulas das turmas $\mathrm{M}$ e $\mathrm{K}$, porém foram devotadas somente quatro aulas ao tema, duas expositivas e duas de resolução de problemas, nos moldes do item anterior.

O processo de análise, do ponto de vista metodológico, procurou levar em conta em primeira instância, o processo de levantamento de todo o tipo de dificuldade que supunhamos que os alunos tivessem encontrado na aprendizagem significativa da Lei de Ampère. Procuramos detectar dificuldades em toda a manifestação exterior do aluno, seja em respostas às questões conceituais, seja na resolução de problemas, seja nas explanações dadas à questão-chave, seja nos desenhos efetuados pelos alunos em suas respostas, seja nas suas declarações. Em uma segunda etapa desse processo, procuramos listar essas dificuldades e verificar quais as mais frequientes, bem como agrupar aquelas que nos pareciam similares, e que variavam apenas na sua forma de apresentação, processo que culminou na categorização das dificuldades, tema do quarto item deste trabalho.

\section{Instrumentos de pesquisa}

Os instrumentos de pesquisa que utilizamos para detectar as dificuldades dos alunos foram de quatro tipos distintos, a saber: questão-chave (aplicada em todas as turmas investigadas), mapa conceitual, questões conceituais e problemas típicos da bibliografia básica adotada em aula (Halliday et al., 1996) envolvendo a Lei de Ampère, aplicados respectivamente a cada turma.

É importante citar que, dos 230 estudantes com quem trabalhamos, nem todos forneceram material necessário e / ou suficiente para análise. Consideramos como casos válidos de estudo 155 estudantes que responderam de forma minimamente aceitável aos instrumentos de pesquisa.

A partir das respostas dadas pelos alunos a essas fontes de investigação, fizemos um levantamento de seus erros mais freqüentes no que se refere à compreensão, interpretação e aplicação da Lei de Ampère, aspecto no qual nos detivemos pela introspecção de que o erro revela a dificuldade que o aluno tem com algum (ou alguns) caráter (es) da lei em questão. Contribuíram de igual forma as declarações dos alunos à questão-chave (na qual se pedia para que os estudantes narrassem suas dificuldades com a lei). Somamos esses dois indicadores para efetuar um primeiro levantamento das dificuldades dos alunos. Verificamos então que, muitas dessas dificuldades tinham núcleos comuns, o que nos permitiu categorizar essas dificuldades em grupos distintos entre si, sendo cada grupo coeso interna- 
mente pela idéia de uma dificuldade comum aos diferentes aspectos em que se manifestava. Como resultado, fizemos uma classificação categorizada das dificuldades dos alunos na aprendizagem significativa da Lei de Ampère, que segue na próxima seção.

\section{Categorização das dificuldades}

Após analisarmos as dificuldades que os alunos expressaram na aprendizagem da Lei de Ampère, caso-a-caso, agrupamos as dificuldades apresentadas mais freqüentes em três categorias distintas, que representam de modo geral as dificuldades dos alunos na aprendizagem significativa da Lei de Ampère, a saber:

a) Funcionalidade da Lei de Ampère;

b) "Superfície" amperiana;

c) Lei de Ampère versus Lei de Faraday.

Os itens acima são genéricos, ou seja, procuram em poucas palavras denotar a dificuldade categorizada. É preciso abordar cada categoria com detalhes para tornar eloqüente o significado do título da mesma. A seguir, faremos esse desdobramento minucioso de cada categoria, a fim de justificála e significá-la, utilizando para isto comentários e exemplos. Trata-se de uma exposição dos dados coletados e de um breve comentário sobre os mesmos. Uma análise superior desses dados será realizada na seção subseqüente deste trabalho.

\section{a) Funcionalidade da Lei de Ampère}

A primeira categoria que nos propomos a analisar é a dificuldade que os alunos apresentam quanto ao entendimento da Lei de Ampère de modo efetivo em relação à natureza do fenômeno, ou seja, interpretar a Lei de Ampère de modo fenomenológico e não como um método. Percebemos que grande parte dos alunos com quem trabalhamos vêem na Lei de Ampère apenas uma maneira prática de calcular o módulo do campo magnético $\mathbf{B}$, sem qualquer outra implicação ou significado físico. Para tais alunos, a Lei de Ampère se restringe a um método de cálculo e obtenção do módulo de $\mathbf{B}$. A asserção de valor fenomenológico que a Lei de Ampère contém, e é a sua razão de ser, que é o fato de tal lei expressar o fenômeno de que uma corrente elétrica cria um campo magnético, é reportada por poucos alunos.

$\mathrm{O}$ aspecto funcional que é dado pelos alunos à Lei de Ampère aparece de duas formas. O modo mais evidente é observado nas declarações dos alunos na resposta à questão-chave, onde os trechos sublinhados endossam esses traços de funcionalidade atribuída à lei:

"A Lei de Ampère é um recurso muito útil no cálculo do campo magnético em situações com elevado grau de simetria." (Cleber)

"O campo magnético criado por um condutor transportando uma corrente pode ser encontrado por esta lei." (Tobias )

"A Lei de Ampère é uma forma de se calcular o campo magnético de uma maneira simetricamente rápida.” ( Leandro )
“A Lei de Ampère é para o cálculo do campo magnético." (Cassiano)

"( a Lei de Ampère) consiste em se escolher um caminho fechado que seja mais adequado ao cálculo do campo magnético criado em determinadas regiões." (Denise)

"(a Lei de Ampère) mede a intensidade do campo magnético nas proximidades de uma superfície de um determinado comprimento." (Pedro)

"A Lei de Ampère serve para resolver problemas com alto grau de simetria, de maneira elegante." (Jorge)

"(a Lei de Ampère) serve para calcular o fluxo magnético dentro de uma região." (Gustavo)

"A Lei de Ampère é o instrumento utilizado para se determinar o B sobre fios fechados, ou espiras.” (Márcio)

Outra espécie de dado que evidencia a funcionalidade da Lei de Ampère se encontra no fato do aspecto restritivo que os alunos têm acerca da Lei de Ampère, que se traduz na dificuldade que os mesmos têm em aplicar a lei aos diversos problemas. O que observamos é que, possivelmente, pelo fato de não terem compreendido satisfatoriamente a Lei de Ampère, o que permitiria aos alunos aplicá-la a uma variedade ampla de casos, os alunos têm uma gama restrita de aplicações da Lei de Ampère, o que faz com que procurem "adequar" um caso clássico de aplicação da mesma (fio longo retilíneo, solenóide, toróide, cabo coaxial) na solução de qualquer problema envolvendo a Lei de Ampère, bem como sempre se restrinjam a estes exemplos, com seu uso permanente, quando solicitados a exemplificar tal lei. Os alunos, ao responderem sobre suas dificuldades, também se referem a esta questão:

"Minha maior dificuldade foi aplicar esta lei em alguns problemas.”(Giovanni )

"Tenho dificuldades em descrever a lei por meio de palavras,sendo mais fácil compreendê-la através de exemplos.”(Carlo)

\section{b) "Superfície" amperiana}

Um aspecto que a Lei de Ampère e a Lei de Gauss partilham em comum é o fato de ambas envolverem, para efeito do cálculo do módulo do campo envolvido (B e $\mathbf{E}$, respectivamente), a utilização de uma figura geométrica que delimita espacialmente o(s) ponto(s) no(s) qual(is) se quer obter o valor do módulo de tal campo.

No caso que enfocamos, que é a Lei de Ampère, sabe-se que o cálculo do campo magnético é efetuado sobre uma $l i$ nha, que é chamada de amperiana. Porém, observamos que muitos alunos se referem à amperiana como uma superfície, e não como uma linha. Este fato aparece sobretudo na questão descritiva da lei. Entretanto, na resolução de problemas, normalmente os alunos calculam o valor de $\mathbf{B}$ fazendo uso de uma integral de linha. Registramos que, geralmente, é na tentativa de fazer uma analogia com a Lei de Gauss (e, por conseguinte, com a superfície gaussiana), que os estudantes falam equivocadamente em superficie amperiana. A esta primeira subcategoria de dificuldades com a Lei de Ampère, no que tange à categoria da dificuldade que chamamos de "superfície" amperiana, denominamos de dificulda- 
des com a terminologia utilizada. As seguintes declarações dos alunos surgem como exemplo de tal manifestação:

“...a Lei de Ampère é calculada sobre uma superfície fechada, chamada superfície amperiana, o círculo na integral diz que ela deve ser calculada em torno de toda a superficie..." (Fernando)

"...o fluxo magnético que passa através de uma superfície amperiana..." (André)

"...assim fazemos uso de uma superfície amperiana fechada, onde desejamos calcular o campo magnético, aplicamos a fórmula e obteremos o campo magnético." (Cristian)

“...fazemos o mesmo para a Lei de Ampère, criamos uma superfície amperiana...” (Leandro)

"Como na Lei de Gauss,coloca-se uma superfície amperiana e calcula-se a integral." (Elton)

Outra espécie de manifestação de dificuldade relacionada à amperiana é o que chamamos de dificuldades em calcular e definir a linha amperiana. Tais dificuldades surgem em duas formas.

A primeira delas ocorre nas declarações dos alunos, que relataram, via questão descritiva, tais obstáculos à amperiana relacionados:

"Minha maior dificuldade foi aceitar a arbitrariedade do percurso de integração." (Claudio)

"A maior dificuldade para a compreensão desta lei é a combinação de percursos (escolha da amperiana).” (Richard )

"A dificuldade para aprender a Lei de Ampère, para mim, é escolher uma amperiana adequada, e também verificar o ângulo entre os vetores, e saber se B é ou não constante sobre ela e pode sair da integral." (Aline)

A segunda forma é expressa nos problemas envolvendo a Lei de Ampère, onde verificamos que vários estudantes não conseguem eleger uma amperiana adequada ao problema em questão.

Não basta escolher uma amperiana adequada, mas, sobretudo, também levar em conta que a amperiana é uma $l i$ $n h a$, não uma superfície. Este erro foi recorrente em alguns alunos, que foram além da questão terminológica supracitada.

Um terceiro manifesto de dificuldade envolvendo o uso da amperiana se encontra na dificuldade com a corrente líquida. Essa espécie de manifestação era por nós esperada, uma vez que, em um trabalho anterior sobre a Lei de Gauss (Krey, 2000), já haviam sido detectados problemas com o conceito de carga líquida. Há dois degraus de apresentação de tal dificuldade. O primeiro está diretamente relacionado com a amperiana, que é basicamente o problema de calcular e definir a linha amperiana, tal qual o citado anteriormente, porém agora estendido ao cálculo de B envolvendo corrente líquida.

Há também alguns alunos que não compreendem o enunciado do problema e / ou não ficam atentos ao mesmo. Por exemplo, um dos problemas apresentados aos alunos é o que trata de uma distribuição de corrente em um fio de raio $\mathrm{R}$, sendo este raio de valor relevante, ou seja, nãodesprezível. Porém, alguns alunos fazem uma errônea interpretação deste fato, considerando o raio do fio como irrelevante, dizendo, assim, que só há campo magnético exterior ao condutor, ou mesmo entendendo que o fio não possui distribuição interna de corrente. A esta segunda forma de apresentação de dificuldade com a corrente líquida, chamamos de dificuldades com a distribuição de corrente.

\section{c) Lei de Ampère versus Lei de Faraday}

Esta é uma dificuldade inerente às turmas $\mathrm{C}$ e H. Nas outras turmas pesquisadas, não houve registros significativos de manifestação de tal dificuldade, possivelmente pelos fatos de:

a) em tais provas não ser solicitada a confecção de mapas conceituais envolvendo alguma das leis em questão (Ampère e Faraday);

b) as provas dessas turmas não conterem questões que abordavam a Lei de Faraday.

A Lei de Faraday, apresentada aos alunos seqüencialmente à lei de Ampère, e fazendo parte do conteúdo da mesma prova em que se obtiveram os resultados em análise, causou confusão a alguns alunos, que a compreenderam, em parte ou todo, como a própria Lei de Ampère. Surgiram manifestações nas respostas dos alunos relacionadas à Lei de Faraday, quando estes eram questionados sobre a Lei de Ampère. Registramos as declarações dos alunos:

"A Lei (de Ampère) nos diz que uma espira ou bobina em movimento dentro de um campo magnético gera corrente nesta espira."(Edemilton )

"A Lei de Ampère nos diz que uma corrente variável produz um campo magnético.”(Juliano)

"A Lei de Ampère nos diz que uma corrente variável produz um campo magnético.

$\oint$ B.ds $=-\mathrm{d} \phi_{E} / \mathrm{dt}$

(Joice)

Uma segunda manifestação desta dificuldade de indistinção entre Leis de Ampère e Faraday ocorre em problemas que envolveram somente a Lei de Ampère. Ao responderem sobre problemas típicos da Lei de Ampère, alguns alunos invocaram elementos pertinentes à Lei de Faraday. Podem ser estas respostas erradas como decorrência de uma desatenção, mas também de uma falta de estabelecimento dos domínios respectivos a cada lei por parte do aluno.

Ocorre que alguns alunos estabelecem uma interferência de conceitos, ao falar em corrente induzida, sabendo-se que a questão é pertinente à Lei de Ampère. Ou seja, é um caso semelhante ao anterior, também refletido nas declarações confusas dos alunos citadas anteriormente, que possivelmente pode ser explicado pela falta de domínios conceituais definidos para cada lei em questão.

Na tabela 1 apresentamos os percentuais de ocorrência de cada categoria e subcategoria de dificuldades que detectamos. Alguns alunos apresentam dificuldades em mais de uma categoria, por isso os percentuais não somam $100 \%$. 
Tabela 1. Ocorrência percentual das categorias e subcategorias das dificuldades detectadas.

\begin{tabular}{|l|l|}
\hline \multicolumn{2}{|l|}{ Categoria: funcionalidade da Lei de Ampère } \\
\hline Subcategoria & Ocorrência percentual* \\
\hline Declarações dos alunos na resposta à questão-chave & 23 \\
\hline $\begin{array}{l}\text { Aspecto restritivo que os alunos têm acerca da lei de } \\
\text { Ampère }\end{array}$ & 28 \\
\hline Categoria: "superfície" amperiana & Ocorrência percentual* \\
\hline Sub-categoria & 15 \\
\hline Dificuldades com a terminologia utilizada & 13 \\
\hline $\begin{array}{l}\text { Escolha equivocada e/ou errônea da amperiana } \\
\text { nos problemas sobre a Lei de Ampère e/ou nas } \\
\text { declarações dos alunos }\end{array}$ & 10 \\
\hline Dificuldade com a corrente líquida & Ocorrência percentual* \\
\hline Categoria: Lei de Ampère versus Lei de Faraday & 16 \\
\hline Sub-categoria & 3 \\
\hline Declarações dos alunos & \\
\hline $\begin{array}{l}\text { Indistinções entre as duas leis, revelada em proble- } \\
\text { mas sobre a Lei de Ampère }\end{array}$ & 3 \\
\hline
\end{tabular}

* Valores percentuais aproximados, calculados sobre a totalidade dos alunos pesquisados.

\section{Discussão dos resultados e recomendações}

Após a apresentação dos resultados obtidos, cabe fazer uma discussão em âmbito particular para cada categoria de dificuldade identificada e, posteriormente, uma discussão dos resultados em âmbito global. Faremos também breves recomendações, que são sugestões nossas para a tentativa de melhoria do processo ensino-aprendizagem no que tange a cada categoria e no aspecto geral da questão.

Primeiramente, faremos comentários acerca de cada categoria em particular, a saber:

\section{a) Funcionalidade da Lei de Ampère}

Havíamos falado, anteriormente, sobre a importância desta categoria de dificuldade, pois ela é o próprio núcleo da questão "dificuldades com a Lei de Ampère", uma vez que ela aborda o conceito da Lei de Ampère. Preocupa-nos, como pesquisadores e professores, o fato que grande parte dos alunos não atribuem um valor fenomenológico real à Lei de Ampère, mas observam na mesma somente um método para cálculo do módulo do campo magnético. O fenômeno que origina a expressão matemática da lei, o fato de que uma corrente elétrica gera um campo magnético, é esquecido pela maioria dos alunos quando convidados a discorrer livremente sobre a Lei de Ampère na resposta à questãochave. Este aspecto fenomenológico é básico para um processo instrucional em que há diferenciação progressiva de conceitos e relações de interação e simetria, uma vez que o fato de que a construção do modelo "corrente elétrica causa campo magnético" é necessário para se incorporar a ele outra proposição, do tipo "campo magnético (variável) causa corrente elétrica (induzida)", e assim por diante, permitindo a construção de um conjunto articulado de proposições no campo do Eletromagnetismo. De fato, observando os outros trabalhos de um projeto mais amplo envolvendo as Equações de Maxwell, chegamos à conclusão de que os alunos têm uma visão desarticulada do Eletromagnetismo, que é visto através de conceitos isolados e sem relação entre si.

Parece-nos que tal visão de funcionalidade da Lei de Ampère vem do processo instrucional a que os alunos são submetidos, uma vez que, a solicitação freqüente de resolução de problemas envolvendo a Lei de Ampère tende a mostrar uma visão muito matematizada da lei, que é então vista como um mero instrumento de cálculo do módulo do campo magnético, em detrimento do aspecto fenomenológico envolvido, que em nosso entender é mais importante que a própria expressão matemática da lei, uma vez que esta é originada pelo fenômeno que ela descreve. Assim, o aspecto físico é deixado em segundo plano para se priorizar o cálculo. Por essa prioridade dada ao cálculo, o aspecto matemático da lei é enfatizado, e a Lei de Ampère é vista então como um método de cálculo, desprovida de um maior significado físico. O próprio fato de se apresentar primeiro aos alunos, no texto e nas aulas, a Lei de Biot-Savart, de maior complexidade matemática, e posteriormente invocar a Lei de Ampère como um modo de se calcular $\mathbf{B}$ com mais simplicidade, endossa o caráter metodológico que é atribuído à Lei de Ampère.

A apresentação dos casos clássicos de aplicação da lei também contribui para que esta seja vista como um método e, de modo ainda mais infeliz, seja vista também sob um ponto de vista restritivo. Claro que isto não deixa de ser verdade, pois o alcance "prático" da Lei de Biot-Savart é mais amplo que o da Lei de Ampère, porém esse fato gera no aluno um processo de sistematização do cálculo de $\mathbf{B}$, onde cada problema proposto, para ser resolvido, é uma questão de adaptação de um caso clássico (fio retilíneo, solenóide, cabo coaxial,...) à solução de tal problema.

Desse modo, os eventuais modelos mentais construídos 
pelo aluno sobre Lei de Ampère ficam muito pobres, e de capacidade restrita para fazer previsões. Ou tornam-se um conjunto de previsões isoladas e desarticuladas entre si, que, a rigor, não constituem modelos mentais. Sem o apelo do fenômeno envolvido, a conexão do conhecimento sobre a Lei de Ampère com as demais equações do Eletromagnetismo torna-se inviável. Alguns alunos parecem ter mentalmente representado o fenômeno, porém como proposição isolada, desvinculada do conjunto do seu conhecimento prévio e posterior sobre Eletromagnetismo.

Tal como na Lei de Gauss (Krey, 2000), os alunos ao estudarem a Lei de Ampère, tendem a representá-la proposicionalmente, ou seja, parecem representá-la simplesmente como proposições do tipo "uma corrente gera um campo magnético", ou "a integral do campo magnético sobre uma linha fechada é igual à corrente"; conseqüentemente, sabendo-se a corrente, calcula-se o campo. A lei serve, então, para calcular o campo magnético.

O problema maior é que este tipo de representação, ainda que possa ser um modelo mental elementar, é muito pobre para dar significado à Lei de Ampère, e mais ainda para utilizá-la para se fazer previsões.

Presumimos que, se o professor priorizasse o ensino da Lei de Ampère do ponto de vista fenomenológico, isto poderia facilitar a aprendizagem significativa da lei por parte do aluno, que assim poderia ter uma visão mais engajada com o significado físico da lei, e menos funcional para com a mesma. A realização de atividades de demonstração experimental da Lei de Ampère concomitantes com a apresentação do conteúdo é sugerida também como aspecto ilustrativo da lei, desvinculando-a do caráter puramente proposicional, e dando a ela uma versão imagística, no sentido dos modelos mentais de Johnson-Laird.

\section{b) "Superfície" amperiana}

Chamamos de "superfície" amperiana, como título genérico, à categoria de dificuldades que estão relacionadas ao conceito de linha amperiana, envolvido na expressão matemática da Lei de Ampère. Esse título é utilizado para nomear esta categoria, pois das dificuldades relacionadas à amperiana, esta é a mais freqüente. São três as subcategorias relacionadas com dificuldades com a amperiana. A primeira delas é justamente essa dificuldade com a terminologia utilizada. Trata-se basicamente de uma questão de terminologia: ao responderem a questão aberta, muitos estudantes falam em superfície amperiana, mas nos problemas fazem o cálculo correto, efetuando uma integral de linha. Alguns confundem os conceitos totalmente, e se dispõem a fazer um cálculo sobre uma superfície; estes, porém, são raros. Isto caracteriza-se como sendo um fato de ordem terminológica, possivelmente causado pela tentativa equivocada de fazer uma analogia entre Lei de Gauss e Lei de Ampère. Gostaríamos de sugerir aos professores que façam uma distinção marcada entre superfície gaussiana e linha amperiana, quando efetuarem tal analogia, para evitar tais equívocos.

Uma segunda subcategoria de dificuldades com a amperiana aborda os problemas que os alunos têm em calcular e definir a linha amperiana. Isto se manifesta em duas formas, uma declarativa e outra operacional.

A primeira forma, que são as declarações dos alunos, é identificada pelas suas respostas à questão-chave. As narrativas dos alunos que se encaixam nesta forma de manifestação pertinente a esta subcategoria relatam seus percalços em compreender o conceito de linha amperiana. Pois é decorrente da compreensão do conceito de amperiana que, em nosso entender, seriam dirimidas as principais dúvidas desses alunos, que questionam a arbitrariedade do percurso de integração, a escolha da amperiana, o vetor ds. Seria aqui nossa recomendação, o fato de se fazer uma explanação sólida do conceito de linha amperiana por parte do professor.

A segunda forma surge nos problemas envolvendo a Lei de Ampère. Constatamos que vários estudantes não fazem uma escolha adequada de amperiana ao problema em questão. Acreditamos que este fato se encontra associado ao aspecto que anteriormente abordamos, que é a nãocompreensão de modo significativo do conceito de linha amperiana. Inferimos então que muitos alunos não constróem um modelo mental satisfatório de linha amperiana, fato deficitário este possivelmente relacionado ao modelo conceitual de linha amperiana que lhes é ensinado.

A terceira subcategoria de dificuldades com a amperiana se encontra na dificuldade com a corrente líquida. Observamos que dois fatores geravam tal manifesto de dificuldade: o problema já conhecido de se lidar de modo adequado com a amperiana e o fato de haver a presença de um termo novo ao aluno - a corrente ponderada. O que registramos é que, em problemas envolvendo corrente líquida com distribuição superficial ou volumétrica de corrente, muitos alunos não conseguem localizar espacialmente a corrente líquida englobada pela amperiana, fazem escolhas de amperianas inadequadas, não sabem ponderar a corrente envolvida pela amperiana ou cometem equívocos quanto à distribuição de corrente. Acreditamos que um modo de reduzir esse tipo de dificuldade seja o fato de se ilustrar com bastante clareza o aspecto da corrente ponderada. Uma vez que o conceito de corrente líquida está vinculado ao de linha amperiana, cremos que à medida que o professor apresenta um modelo conceitual claro de amperiana, ele está contribuindo para uma melhor compreensão, por parte do aluno, do tema "corrente líquida".

\section{c) Lei de Ampère versus Lei de Faraday}

Um fato que, de certa forma, torna-se surpreendente, é esta categoria de dificuldades apresentada pelos alunos. A Lei de Faraday é o conteúdo apresentado aos alunos imediatamente após o tópico sobre a Lei de Ampère. Vários alunos, respondendo a questões sobre a Lei de Ampère, se reportam a elementos característicos do domínio da Lei de Faraday, fazendo uma possível, parcial ou total, indistinção entre as duas leis.

Esta dificuldade se verificou somente nas turmas pesquisadas "C" e "H", em cujas provas analisadas haviam questões e problemas sobre tais leis, porém separadamente. Nas provas das demais turmas, tal evidência não foi regis- 
trada; nelas, havia questões abordando somente a Lei de Ampère. Talvez, observando-se as provas que abordaram a Lei de Faraday, se registrassem indícios desta evidência, porém, quando a detectamos, já não dispúnhamos da possibilidade de ter acesso a tais provas. Contribui também para que tenham sido detectados tais indícios, nas turmas " $\mathrm{C}$ " $\mathrm{e}$ "H", o fato de que nas provas de tais turmas era solicitada a confecção de um mapa conceitual da Lei de Ampère e outro sobre a Lei de Faraday. Os alunos das turmas "C" e "H" já haviam visto as duas leis em aula quando da prova realizada, e os alunos das demais turmas pesquisadas apenas tomaram contato com a Lei de Faraday após a realização da prova envolvendo a Lei de Ampère.

Ressaltando-se ainda o fato de que tais indícios de confusão entre a Lei de Ampère e a Lei de Faraday não ocorrem somente nas questões abertas e nos mapas conceituais, mas também na resolução de problemas, é que atentamos ainda mais às sérias implicações que esta dificuldade tem do ponto de vista do ensino dessas duas leis.

Presumimos que há uma questão semântica que acentua a confusão entre as duas leis, que se situa em torno do significado da palavra variação. A palavra "variação" tem um único sentido no que se refere à Lei de Faraday: campo magnético variável produz corrente elétrica. Alguns alunos pensam que deve haver reciprocidade quando se fala em Lei de Ampère, ou seja, acreditam que apenas uma corrente variável possa gerar um campo magnético. Sugerimos que professores devem fazer um esclarecimento aos alunos sobre esta questão para evitar esse tipo de equívoco. O mesmo se aplica à dificuldade que os alunos têm em falar em corrente induzida ao abordar questões sobre a Lei de Ampère, que é certamente não apenas uma questão gramatical, mas, mais do que isso, uma questão de definição de domínios quanto ao espectro conceitual de cada lei em questão.

Após termos realizado uma discussão dos resultados para cada categoria, faremos algumas considerações gerais sobre os resultados analisados.

Inferimos, supomos, que, a rigor, nenhum dos alunos pesquisados formou um modelo mental propriamente dito da Lei de Ampère a partir das respostas coletadas. Nenhum dos alunos manifestou domínio imagístico do tema, alguns deram evidências de um modelo proposicional um pouco elaborado, mas a grande maioria parecem ser proposicionalistas de sentido bastante restrito, ou seja, vêem a Lei de Ampère por meio de proposições isoladas e desarticuladas entre si.

Queremos salientar que, em nossa visão, duas das categorias de dificuldade identificadas ("superfície" amperiana e Lei de Ampère versus Lei de Faraday) estão associadas à visão integradora dos conceitos do Eletromagnetismo, e poderá ser minorada a freqüência com que elas ocorrem se o professor agir de modo a integrar e inter-relacionar conceitos envolvidos. Por exemplo, uma analogia bem efetuada, que faça distinções marcadas entre superfície gaussiana e linha amperiana, definindo bem esses conceitos e seus domínios, além de vincular esses conceitos, os distinguirá, evitando a confusão entre os mesmos. O mesmo se dá entre os domínios das leis de Ampère e Faraday: esses fenômenos devem ser vistos de modo vinculado dentro do conjunto do Eletromagnetismo, e possíveis analogias, se houverem, podem até serem exploradas, porém é necessário distinguir bem seus domínios e suas aplicações.

Acrescentamos ainda que, uma ênfase fenomenológica do tema "Lei de Ampère" pode colaborar para reduzir tais dificuldades, reforçando conceitos e dando uma visão menos funcional de tal lei. Experimentos simples, realizados em aula pelos alunos ou em demonstrações feitas pelo professor, como, por exemplo, a experiência de Oersted (agulha imantada da bússola defletida por influência da passagem de corrente elétrica num fio condutor próximo a ela) seriam recomendáveis para tanto.

As dificuldades apontadas — a lei vista como um método, a escolha da amperiana, o cálculo da corrente líquida e a confusão entre as Leis de Ampère e Faraday são em grande parte decorrentes do modo de ensino apresentado ao aluno e da maneira como o tema é enfocado pelo livro-texto. Foi em função disso que fizemos algumas recomendações aos professores enquanto comentávamos tais dificuldades, porém a questão é bem mais profunda, pois envolve modelagem mental. Segundo Johnson-Laird, o modelo mental é o principal instrumento humano de compreensão; quando compreendemos algo (notadamente no sentido de saber descrever, explicar e fazer previsões sobre esse algo) é porque foi construído em nossa memória de trabalho um modelo mental da situação. Por exemplo, quando compreendemos o que é um solenóide, isto ocorre porque construímos em nossa mente um análogo estrutural desse dispositivo, em suma, um modelo mental dele. Da mesma forma, quando entendemos que cargas em repouso geram um campo elétrico, e quando tais cargas se movimentam surge também um campo magnético, é porque fizemos mentalmente um modelo de como isso ocorre, ou seja, um modelo mental de tais fenômenos.

De modo análogo, para compreender, para aprender significativamente, a expressão $\oint \mathbf{B}$.dl $=\mu_{0} i$, é preciso construir um modelo mental dessa expressão. É necessário entendê-la como uma lei geral do Eletromagnetismo, e não como uma fórmula. Quando tal aprendizagem significativa acontece, os casos clássicos como o do fio retilíneo infinito ou do cilindro (oco ou maciço) passam a se tornar vistas do modelo, imagens do modelo. Desse modo, ainda que não seja possível ter uma "visão geral" da lei, é possível imaginar o que ocorrerá em uma dada situação, com esse ou aquele campo magnético, com essa ou aquela corrente elétrica.

Naturalmente, para se compreender significativamente a amperiana, também é necessária a construção de um modelo mental tal que cada caso particular de linha amperiana seja visto como uma perspectiva desse modelo. Assim, aquele que aprende de modo significativo o que é uma amperiana, via construção de um modelo mental da mesma, conhece seus atributos e irá distingui-la de uma gaussiana. Entretanto, não poderá imaginar uma amperiana em geral, e sempre que recorrer a tentar imaginá-la, virá à sua mente a 
imagem de uma particular amperiana.

Na mesma linha de raciocínio, a compreensão do que vem a ser corrente líquida, implica também construir um adequado modelo mental desse conceito, que permitirá identificar casos de soma algébrica e de ponderação da (s) corrente (s) envolvida (s) pela amperiana.

Torna-se evidente então que, para uma satisfatória modelagem mental da Lei de Ampère, é necessária a modelagem prévia de conceitos-chave pertinentes, o que significa que um modelo mental da Lei de Ampère terá "submodelos".

Porém, não podemos esquecer que a modelagem mental da Lei de Ampère é feita a partir da percepção que o aluno tem do modelo conceitual, em geral um modelo matemático do modelo físico da situação física (o campo magnético gerado por uma corrente elétrica).

Em suma, a compreensão significativa de uma lei tal como a Lei de Ampère requereria um ensino bastante diferente daquele que os alunos aqui pesquisados vivenciaram. Seria preciso um ensino que ajudasse os alunos a modelar a lei mentalmente e, em conseqüência, permitisse modelar a situação física envolvida, o que certamente viria a facilitar a resolução de problemas envolvendo essa lei. Porém, não é isso o que ocorre: a lei é simplesmente apresentada ao aluno e, a seguir, já se parte para a sua aplicação em problemas. Sequer é devotada atenção ao crucial fato de que é necessário saber como é o campo magnético para então escolher uma amperiana que permita resolver facilmente a integral de linha e então, por fim, obter a intensidade do campo magnético.

Conforme dissemos anteriormente, a Lei de Ampère deveria ser introduzida através do fenômeno eletromagnético básico que ela descreve, porém isso não seria suficiente. Seria necessário também ensinar ao aluno técnicas de modelagem física e abstração, por exemplo, que o auxiliassem a construir modelos mentais que pudessem dar significado ao modelo conceitual que lhes foi ensinado, no caso, a Lei de Ampère.Tais modelos poderiam evoluir para esquemas de assimilação que o aluno acionaria para lidar com situações que envolvessem a Lei de Ampère.

Entretanto, isso constituiria trabalho para outra pesquisa, certamente muito mais complexa. Nesta, nos propusemos apenas a identificar as dificuldades dos alunos e a interpretálas à luz da Teoria dos Modelos Mentais de Johnson-Laird ${ }^{1}$. Acreditamos ter alcançado esse objetivo, pois vislumbramos três categorias de dificuldades, as quais nos parecem ilustrar bastante bem qual a dificuldade maior dos alunos: a construção de um modelo mental da Lei de Ampère. Os alunos não vão além de percebê-la como um método, ou uma fórmula, para resolver determinado tipo de problema. $\mathrm{Na}$ verdade, isso não é surpreendente no contexto do ensino da Física: os alunos tendem a ver a Física como uma ciência "de fórmulas". Esperamos que este trabalho possa servir como um alerta de que são necessárias mudanças profundas no ensino da Física, se nosso intuito é, de fato, querermos uma aprendizagem significativa e não mecânico-formulista dessa Ciência.

\section{Referências}

[1] GENTNER, D., STEVEnS, A. L. (Eds.) Mental Models. Hillsdale, NJ: Lawrence Erlbaum Associates, 1983.

[2] HALLIDAY, D., RESNICK, R., WALKER, J. Fundamentos de Física. 4. ed. Rio de Janeiro: LTC, 1996. v. 3.

[3] JOHNSON-LAIRD, P. N. Mental models. Cambridge: Cambridge University Press, 1983.

[4] KREY, I. Dificuldades dos alunos na aprendizagem da lei de Gauss à luz da teoria dos modelos mentais de JohnsonLaird. 2000. 99f. Dissertação (Mestrado em Física)-Instituto de Física, Universidade Federal do Rio Grande do Sul, Porto Alegre, 2000.

[5] MOREIRA, M. A. Modelos Mentais. Investigações em Ensino de Ciências, Porto Alegre, v. 1, n. 3, p. 95-108, dez. 1996.

[6] NORMAN, D. A. Some observations on mental models. In: GENTNER, D.; STEVENS, A. L. (Eds.) Mental models. Hillsdale: Lawrence Erlbaum Associates,1983. p.6-14.

[7] POZO, J. I. Mas allá del cambio conceptual: el aprendizaje de la ciencia como cambio representacional. Enseñanza de las Ciencias, v. 17, n. 3, p. 513 - 520, Mar.1999.

\footnotetext{
${ }^{1}$ Registre-se que em nenhum momento tentamos identificar modelos mentais construídos pelos alunos, apenas inferimos que, provavelmente, eles não chegaram a construir tais modelos.
} 